\title{
Avaliação Psicométrica do Male Body Checking Questionnaire (MBCQ)
}

\section{Psychometric Assessment of the Male Body Checking Questionnaire (MBCQ)}

\author{
Pedro Henrique Berbert de Carvalho*, ${ }^{a}$, Maria Aparecida Conti ${ }^{b}$, Mário Sérgio Ribeiro $^{c}$, \\ Ana Carolina Soares $\mathrm{Amaral}^{d} \&$ Maria Elisa Caputo Ferreira ${ }^{c}$ \\ ${ }^{a}$ Universidade Federal de Juiz de Fora, Governador Valadares, MG, Brasil, \\ ${ }^{b}$ Universidade de São Paulo, SP, São Paulo, Brasil \\ ${ }^{c}$ Universidade Federal de Juiz de Fora, Juiz de Fora, MG, Brasil \\ \& Instituto Federal do Sudeste de Minas Gerais, Barbacena, MG, Brasil
}

\begin{abstract}
Resumo
Este trabalho teve como objetivo avaliar as propriedades psicométricas do Male Body Checking Questionnaire (MBCQ), com recurso à análise fatorial exploratória e confirmatória, validade convergente e discriminante, e reprodutibilidade (coeficiente de correlação intraclasse e $\alpha$-Cronbach), realizadas com 287 universitários brasileiros entre 18 e 30 anos de idade. O MBCQ obteve estrutura fatorial adequada, com quatro fatores que explicam $64,32 \%$ da variância dos resultados, validade convergente - associação significativa $(p<0,01)$ com a insatisfação e checagem corporal ( $r$ igual a 0,51 e 0,35 , respectivamente), e discriminante. Obteve satisfatória consistência interna ( $\alpha$-Cronbach variando de 0,89 a 0,94 ) e coeficiente de correlação intraclasse de 0,87 . O MBCQ comprovou ser um instrumento válido e confiável para avaliação da checagem corporal para indivíduos do sexo masculino do Brasil.

Palavras-chave: Psicometria, checagem corporal, validade, análise fatorial.
\end{abstract}

\begin{abstract}
This study aimed to analyze the psychometric properties of the Male Body Checking Questionnaire (MBCQ) applying exploratory and confirmatory factor analysis, concurrent and discriminant validity, and reliability (intra-class coefficient correlation and Cronbach's alpha). Two hundred and eighty seven Brazilian undergraduate students between 18 and 30 years old were evaluated. The MBCQ showed adequate factor structure with four factors that explain $64.32 \%$ of total variance, concurrent validity - significant association with body dissatisfaction $(p<.01)$ and body-checking $(r=.51$ and .35 , respectively), and discriminant validity. The MBCQ also showed good internal consistence (Cronbach's $\alpha$ between .89 and .94) and intra-class correlation coefficient (.87). In conclusion, it is a valid and reliable instrument to evaluate body-checking behaviors of Brazilian males.

Keywords: Psychometrics, body-checking, test validity, factor analysis.
\end{abstract}

A checagem corporal é um comportamento comum e de importância clínica para os transtornos alimentares (TA). Incluem atitudes com o intuito de obter informações sobre o peso, o tamanho e a forma corporal, por meio de ações constantes de aferição do peso, "pinçamento" da gordura e pele, visualização do corpo em superfícies reflexivas, e comparação de seu corpo com o de outros indivíduos (Shafran, Fairburn, Robinson, \& Lask, 2004).

A checagem corporal, bem como a superestimação do peso e da forma corporal compõem um dos aspectos psicopatológicos centrais relacionados aos TA (Reas, Grilo, Masheb, \& Wilson, 2005; Shafran et al., 2004).

\footnotetext{
* Endereço para correspondência: Universidade Federal de Juiz de Fora. Faculdade de Educação Física e Desportos. Rua José lourenço Kelmer, s/n, Campus Universitário, Bairro São Pedro, Juiz de Fora, MG, Brasil. 36036-900. E-mail: pedro.berbert@gmail.com
}

Sua frequência está associada diretamente à severidade do quadro em questão, sendo que pacientes diagnosticados com anorexia nervosa, bulimia nervosa e transtorno de compulsão alimentar periódica demonstram alta frequência desta atitude (Shafran et al., 2004). Ademais, este comportamento se associa, também, a crenças disfuncionais dos pacientes como, por exemplo, as de que a checagem serve para manter o controle sobre a alimentação e o peso, diminui a ansiedade, e que traz ajuda para sentirem-se melhor (Mountford, Haase, \& Waller, 2006).

O comportamento de checagem corporal pode ser visto, ainda, como um fator na manutenção de aspectos psicopa- tológicos dos TA (Fairburn, Shafran, \& Cooper, 1999; Williamson, Muller, Reas, \& Thaw, 1999). Ao recorrer a monitoramento constante do corpo (checagem corporal), pacientes com TA procuram avaliar sua eficácia no controle do peso. Em caso de interpretação de fracasso sobre o controle de seu corpo, pacientes com TA reforçam 
a adoção de medidas inadequadas, como, por exemplo, a restrição alimentar, e expressam o aumento da insatisfação corporal (Fairburn et al., 1999; Shafran, Lee, Payne, \& Fairburn, 2007).

Assim, ações no sentido de diminuir o comportamento de checagem corporal são alvos do tratamento de pacientes com TA (Fairburn, 2008) e transtorno dismórfico corporal (Rosen, Reiter, \& Orosan, 1995). Essas ações são também apontadas como importante forma de intervenção para os distúrbios de imagem corporal (Cash \& Smolak, 2011; Exterkate, Vriesendorp, \& De-Jong, 2009; Stice \& Shaw, 2002).

Os TA, por exemplo, são transtornos psicopatológicos de identificação recente para indivíduos do sexo masculino e oferecem um grande desafio, como sua descrição clínica e nosológica (Murray et al., 2012). Apesar de apresentarem os mesmos critérios diagnósticos de TA que as mulheres (American Psychiatric Association [APA], 1994), os homens demonstram um quadro mais complexo no que se refere às preocupações com o corpo. Assim como as mulheres, indivíduos do sexo masculino preocupam-se com o peso corporal e o percentual de gordura, no entanto, em adição apresentam elevado desejo de atingir um corpo musculoso, especialmente na região superior do tronco (peitorais, ombros e braços; Pope, Phillips, \& Olivardia, 2000; Thompson \& Cafri, 2007).

A fim de avaliar a frequência dos comportamentos de checagem corporal, Hildebrandt, Walker, Alfano, Delinsky e Bannon (2010) desenvolveram o Male Body Checking Questionnaire (MBCQ), baseado em um dos instrumentos mais utilizados para a avaliação da checagem corporal, o Body Checking Questionnaire (BCQ; Reas, Whisenhunt, Netemeyer, \& Williamson, 2002). No entanto, essa versão foi modificada para atender às particularidades da população masculina (Hildebrandt et al., 2010).

No estudo original de validade (Hildebrandt et al., 2010), o MBCQ confirmou sua validade, apresentando associação positiva e significativa $(p<0,01)$ com sintomas de perfeccionismo, de transtorno alimentar e da dismorfia muscular; e também com comportamentos de checagem corporal (indicadores de validade convergente). O MBCQ foi igualmente capaz de discriminar indivíduos com comportamentos de purgação e/ou de compulsão alimentar daqueles sem esses comportamentos (Hildebrandt et al., 2010). No que diz respeito a sua estrutura interna, foram identificados, por meio de análise fatorial, quatro fatores que responderam por $66,91 \%$ da variância total do instrumento: (a) Global Muscle Checking (GMC) - Checagem Muscular Global, (b) Chest and Shoulders Checking (CSC) - Checagem de Peito e Ombro, (c) Other-Comparative Checking (OCC) - Outros - Checagem Comparativa, e (d) Body Testing (BT) - Checagem Corporal. A consistência interna foi adequada para todos os itens que compõem o instrumento ( $\alpha$-Cronbach superior a 0,72 ), assim como para cada fator $(\mathrm{GMC}=0,86 ; \mathrm{CSC}=0,85 ; \mathrm{OCC}=0,83$; $\mathrm{BT}=0,72$; Hildebrandt et al., 2010).
Os autores do estudo original, já referido, sugerem a utilização da escala especialmente para sujeitos do sexo masculino, uma vez que o modelo da medida não se ajustou para sujeitos do sexo feminino. Segundo Hildebrandt et al. (2010), os resultados reforçam a necessidade da aplicação de escalas específicas a cada sexo, uma vez que a checagem corporal pode ser fortemente influenciada por essa variável e, portanto, pode estar associada a aspectos da aparência diferencialmente valorizados entre mulheres e homens (por exemplo, magreza versus muscularidade).

A avaliação prévia do MBCQ foi realizada para jovens adultos brasileiros (18 a 26 anos de idade), por meio das análises de equivalência semântica e da consistência interna. Foram realizadas: tradução, retrotradução, síntese de tradução, avaliação do comitê de peritos, pré-teste, e análise da consistência interna (Carvalho, Conti, Cordás, \& Ferreira, 2012). O instrumento apresentou boa compreensão por parte dos especialistas e estudantes (equivalência semântica) e obteve resultados satisfatórios de consistência interna para o instrumento como um todo $(\alpha$-Cronbach de $0,96)$ e para os quatro fatores que o compõe: Checagem Muscular Global ( $\alpha$-Cronbach de 0,92), Checagem de Peito e Ombro ( $\alpha$-Cronbach de 0,85 ), Outros - Checagem Comparativa ( $\alpha$-Cronbach de 0,83 ), e Checagem Corporal $(\alpha$-Cronbach de 0,90$)$.

No Brasil, há alguns instrumentos específicos para a avaliação dos comportamentos de checagem corporal, como: Body Checking Questionnaire (Campana, 2007); Body Checking Cognition Scale (Kachani, Barbosa, et al., 2011) e o Body Checking and Avoidance Questionnaire (Kachani, Hochgraf, et al., 2011), mas todos são específicos para sujeitos do sexo feminino. Até o momento nenhum instrumento foi submetido a uma avaliação de suas qualidades psicométricas (estrutura fatorial, validade convergente e discriminante) para comprovar um modelo de medida dos comportamentos de checagem corporal de sujeitos do sexo masculino. A disponibilidade de tal instrumento poderá ser útil como ferramenta de avaliação na área clínica e epidemiológica. Assim, esta pesquisa teve como objetivo avaliar as qualidades psicométricas do Male Body Checking Questionnaire (MBCQ) para jovens adultos do sexo masculino do Brasil.

\section{Método}

\section{Participantes}

Participaram 287 indivíduos do sexo masculino, com idade entre 18 e 30 anos $(M=21,27 ; D P=2,71)$, e Índice de Massa Corporal (IMC) entre 16.37 e $47.34 \mathrm{~kg} / \mathrm{m}^{2}(M=$ 23,$63 ; D P=3,49$ ), derivado do peso e estatura autorrelatados. Os participantes eram alunos universitários dos cursos de graduação (Ciências da Computação, Direito, Engenharia Civil, Engenharia Elétrica, Filosofia, Fisioterapia, Matemática, Medicina e Psicologia) da Universidade Federal de Juiz de Fora, Minas Gerais, Brasil, selecionados 
por conveniência, de acordo com a presença em sala de aula e disponibilidade para o preenchimento dos questionários. Não foram incluídos sujeitos dos cursos de Educação Física e Nutrição por sabidamente apresentarem elevada preocupação com o corpo, além de comportamentos alimentares inadequados (Bosi, Luiz, Uchimura, \& Oliveira, 2008; Fiates \& Salles, 2001), visto que são variáveis que podem influenciar o construto em análise.

\section{Instrumentos}

Male Body Checking Questionnaire (MBCQ; Hildebrandt et al., 2010). O MBCQ é um instrumento de autorrelato, em formato de escala Likert de 5 pontos (1 - Nunca a 5 - Muito frequentemente), composto por 19 itens, e destinado à avaliação dos comportamentos de checagem corporal de jovens adultos. Foi utilizada a versão traduzida e adaptada para a língua portuguesa do Brasil, em sua forma completa (Carvalho et al., 2012). Para o cálculo do escore total são somadas as pontuações de cada item e a pontuação varia de 19 a 95 pontos. Embora não exista um ponto de corte para se considerar como índice clínico, quanto maior a pontuação, mais frequentes são os comportamentos de checagem corporal (Hildebrandt et al., 2010). O MBCQ é composto por quatro fatores: Checagem Muscular Global (itens: 1, 2, 3, 4, 5, 14 e 15); Checagem de Peito e Ombro (itens: 11, 12 e 13); Outros - Checagem Comparativa (itens: 6, 7, 8 e 9); e Checagem Corporal (itens: 10, 16, 17, 18 e 19).

Body Checking Questionnaire (BCQ; Reas et al., 2002). O BCQ é um instrumento de autorrelato composto por 23 itens, em formato de escala Likert de 5 pontos (1 - Nunca a 5 - Muito frequentemente), destinado à avaliação da checagem corporal de mulheres adultas. $\mathrm{O}$ escore total é calculado pela soma da pontuação de cada item. Quanto maior o escore total, maior é a frequência dos comportamentos de checagem corporal. A versão brasileira (Campana, 2007) foi utilizada nesse trabalho e é formada por quatro fatores: checagem pela observação do corpo (OB), checagem por meio de medições de partes do corpo (ME), checagem com comparações entre o corpo do sujeito e os corpos de outros (CO) e busca por informações perceptivas (IP). O BCQ foi utilizado para avaliação da validade convergente do MBCQ, sendo esperada uma correlação significativa positiva com o MBCQ. Foi calculada a consistência interna, por meio do coeficiente $\alpha$-Cronbach, para os dados do BCQ da amostra deste estudo, que correspondeu a 0,76 .

Body Shape Questionnaire (BSQ; Cooper, Taylor, Cooper, \& Fairburn, 1987). O BSQ é um instrumento de autorrelato composto por 34 itens, em formato de escala Likert de 6 pontos $(1-$ Nunca a 6 - Sempre $)$, utilizado para avaliação das preocupações quanto ao peso e a forma corporal. O escore total é obtido pela soma dos escores de cada item. Quanto maior a pontuação, maior a insatisfação do indivíduo com seu corpo (Cooper et al., 1987). A versão brasileira foi validada para ambos os sexos, apresentando satisfatória consistência interna ( $\alpha$-Cronbach de 0,97; Di Pietro \& Silveira, 2009). O BSQ foi utilizado no presente trabalho para avaliação de possíveis evidências de validade convergente com o MBCQ. Segundo Fairburn et al. (1999) a insatisfação e a checagem corporal estão diretamente associadas, ou seja, quanto maior a insatisfação com o corpo, maior é a frequência de checagem. Assim, espera-se que os escores obtidos no MBCQ e o BSQ apresentem associação positiva significativa. A consistência interna do BSQ, calculada a partir dos resultados da amostra do presente estudo, mostrou-se adequada $(\alpha$-Cronbach $=0,92)$.

Eating Attitudes Questionnaire - 26 (EAT-26; Garner, Olmsted, Bohr, \& Garfinkel, 1982). O EAT-26 é um instrumento de autorrelato composto por 26 itens, em formato de escala Likert de 3 pontos ( 0 - Nunca, Raramente e Muito raramente $;=$ Frequentemente $; 2=$ Muito frequentemente; 3 = Sempre), destinado à avaliação de sintomas relacionados à alteração da atitude alimentar. No EAT-26 o escore superior a 20 pontos indica presença de atitudes alimentares patológicas e risco para o desenvolvimento de transtorno do comportamento alimentar (Garner et al., 1982). A versão brasileira, de acordo com Nunes, Camey, Olinto e Mari (2005), apresenta boas propriedades psicométricas, com satisfatória consistência interna $(\alpha$-Cronbach de 0,75$)$. O EAT-26 foi aplicado no presente estudo com a finalidade de permitir exame de evidências de validade discriminante em relação ao MBCQ. Como apontado por Shafran et al. (2004), indivíduos com TA apresentam frequência significativamente maior dos comportamentos de checagem corporal quando comparados a indivíduos saudáveis. Portanto, é esperada diferença significativa na pontuação do MBCQ entre os grupos de baixa e alta presença de atitudes alimentares patológicas. A consistência interna do EAT-26, calculada a partir dos resultados da amostra do presente estudo, mostrou-se adequada $(\alpha$-Cronbach $=0,72)$.

\section{Procedimentos}

Após contato com os coordenadores de curso e professores, os sujeitos da pesquisa foram abordados em grupos, no ambiente de sala de aula e durante os horários das aulas. Estes receberam informações sobre o objetivo da pesquisa e os procedimentos a serem seguidos, e assegurado o anonimato de cada participante. Todos assinaram o Termo de Consentimento Livre e Esclarecido, autorizando sua participação voluntária. Em seguida, os participantes responderam individualmente aos instrumentos propostos na seguinte ordem: MBCQ, BCQ, BSQ e EAT-26. Não foi dada informação adicional às instruções já existentes em cada instrumento proposto. Em caso de dúvida os participantes eram orientados e ler novamente cada item do questionário, buscando uma compreensão individual sobre o item proposto. Os indivíduos reportaram ainda sua idade e, também, sua massa corporal e estatura, que foi utilizada para o calculado 
Carvalho, P. H. B., Conti, M. A., Ribeiro, M. S., Amaral, A. C. S. \& Ferreira, M. E. C. (2014). Avaliação Psicométrica do Male Body Checking Questionnaire (MBCQ).

o Índice de Massa Corporal (IMC), dado pela massa corporal (quilogramas) dividida pela estatura (metros) elevada ao quadrado.

Para análise da reprodutibilidade teste-reteste do MBCQ, foi selecionada aleatoriamente uma turma para a reaplicação do instrumento, com intervalo de duas semanas entre as aplicações (Thompson, 2004). Participaram dessa fase do estudo 32 indivíduos com média de idade de 19,84 $(D P=2,18)$ anos e com IMC de $23.42(D P=3,17) \mathrm{kg} / \mathrm{m}^{2}$. Não houve diferença estatisticamente significativa para a média de idade e IMC entre os indivíduos participantes do reteste e da amostra total.

Esta pesquisa está de acordo com os princípios da Declaração de Helsinki, sua execução está de acordo com as normas da Resolução n ${ }^{\circ}$. 196/96 do Conselho Nacional de Saúde, e foi aprovada pelo Comitê de Ética em Pesquisa com Seres Humanos da Universidade Federal de Juiz de Fora (Protocolo n ${ }^{\mathrm{o}}$ 2.193.253.2010).

\section{Análise dos Dados}

Foi realizada análise descritiva dos dados (média, desvio-padrão, valor mínimo e máximo) dos escores obtidos dos instrumentos MBCQ, BCQ, BSQ e EAT-26, e também da idade e IMC dos participantes. Em adição, foi realizado cálculo do coeficiente $\alpha$-Cronbach para averiguar a consistência interna de todos os instrumentos supracitados. Valor superior a 0,70 foi considerado satisfatório (Streiner, 2003). Para essas análises e Análise Fatorial Exploratória (AFE) do MBCQ foi utilizado o software SPSS v.17.0, adotando o nível de significância de 5\%.

Foi realizado teste de normalidade Kolmogorov-Smirnov dos dados obtidos nos instrumentos utilizados (MBCQ, BCQ, BSQ, EAT-26) que indicou normalidade em sua distribuição. As evidências de validade convergente foram avaliadas adotando-se análise estatística paramétrica, recorrendo-se a análises de correlação de Pearson entre os escores dos instrumentos MBCQ e BCQ, e também, MBCQ e BSQ.

As evidências de validade discriminante foram averiguadas pela comparação dos escores totais obtidos do MBCQ, por meio do teste $t$ de Student de medidas independentes, entre indivíduos com baixo e elevado escore de sintomas de TA, avaliado pelo EAT-26. Segundo Hildebrandt et al. (2010) o MBCQ é capaz de discriminar indivíduos com sintomas relacionados à alteração da atitude alimentar daqueles sem esses sintomas. Como, em adição a amostra de universitários não foram avaliados indivíduos diagnosticados com TA, recorreu-se ao EAT26. Utilizou-se os pontos de corte propostos por Orbitello et al. (2006) para categorizar indivíduos com baixo (EAT-26 $<11$ pontos) e elevado (EAT-26 $\geq 11$ pontos) sintomas de alteração da atitude alimentar.

A reprodutibilidade (teste-reteste) do MBCQ foi avaliada pela comparação entre os escores obtidos (recorrendo-se ao teste de $t$ de Student, de medidas emparelhadas), com intervalo de duas semanas entre os dois momentos avaliados, e pelo coeficiente de correlação intraclasse. Além disso, foi verificada a consistência interna, por meio do cálculo de coeficiente $\alpha$-Cronbach, nos dois momentos (Hair, Tatham, Anderson, \& Black, 2005).

O MBCQ preencheu os critérios padronizados para normalidade multivariada (coeficiente de Mardia próximo de zero). Não foi observada inclinação maior do que três ou curtose maior que dez, de modo que não foi necessária a transformação logarítmica anterior à realização da análise fatorial (Kline, 1998).

$\mathrm{Na}$ Análise Fatorial Exploratória (AFE) do MBCQ, realizada pelo método de componentes principais, seguido de uma rotação ortogonal Varimax, adotou-se o critério Kaiser (eigenvalue mínimo de 1) para a extração dos fatores (Hair et al., 2005). O teste de esfericidade de Bartlett e a medida Kaiser-Meyer-Olkin (KMO) foram aferidos para atestar a adequação dos dados à análise fatorial. A matriz das cargas fatoriais foi analisada para identificação dos itens e suas correspondências com os fatores. Valores superiores a 0,5 foram considerados como significativos (Kahn, 2006). O coeficiente $\alpha$-Cronbach foi avaliado em cada fator identificado, a fim de averiguar a consistência interna dos mesmos. Os fatores foram, então, nomeados de acordo com as dimensões latentes que representavam, ou seja, de acordo com o conteúdo ao qual cada fator se destina avaliar (Hair et al., 2005).

A Análise Fatorial Confirmatória (AFC) foi utilizada para avaliar a estrutura fatorial do modelo obtido na AFE. Foram utilizadas as seguintes medidas de ajustes: Qui-quadrado Ponderado $\left(\chi^{2}\right)$, Comparative Fit Index (CFI), Tucker-Lewis Index (TLI), Root Mean Square Error of Approximation (RMSEA), Akaike Information Criterion (AIC - Independence e Model), Standardized Root Mean Square Error of Approximation (SMSEA), Standardized Root Mean Square Residual (SRMR) e Incremental Fit Index (IFI) (Kahn, 2006; Kline, 1998).

As análises da AFC foram realizadas no software LISREL, v.8.8. Foram considerados bons ajustes: $\chi^{2}$ não significativo, valores superiores a 0,9 para CFI, TLI e IFI (Bentler \& Bonett, 1980); inferiores a 0,08 e 0,06 para RMSEA e SRMR, respectivamente (Kahn, 2006); e o valor obtido no AIC Model menor do que o AIC Independence (Akaike, 1987).

\section{Resultados}

\section{Análise Descritiva}

Os escores obtidos e as correlações entre os instrumentos estão resumidos na Tabela 1. Destaca-se que os instrumentos utilizados apresentaram uma importante variação nos escores obtidos pelos sujeitos da pesquisa, compreendendo, portanto, quase a totalidade de variação do escore possível em cada instrumento (de escore mínimo ao escore máximo). Variação máxima foi observada somente para o MBCQ (19 a 95 pontos). O escore médio foi de $33.43(D P=11,78)$ pontos nesse instrumento. Embora não exista uma categorização dos escores do instrumento, observa-se que o escore médio somado a um desvio-padrão 
Psicologia: Reflexão e Crítica, 27(4), 700-709.

Tabela 1

Análise Descritiva dos Dados e Correlação dos Instrumentos

\begin{tabular}{lrrrrrrrr}
\hline Variáveis & $M$ & \multicolumn{1}{c}{$D P$} & Mínimo & Máximo & 1 & 2 & 3 & 4 \\
\hline 1. MBCQ & 33,43 & 11,78 & 19,00 & 95,00 & - & & & \\
2. BCQ & 16,36 & 4,42 & 12,00 & 40,00 & $0,51^{*}$ & - & & \\
3. BSQ & 55,47 & 17,35 & 34,00 & 135,00 & $0,35^{*}$ & $0,59^{*}$ & - & \\
4. EAT-26 & 7,16 & 4,55 & 0,00 & 20,00 & $0,18^{*}$ & $0,33^{*}$ & $0,48^{*}$ & - \\
\hline
\end{tabular}

Nota. 1 - MBCQ. Male Body Checking Questionnaire; 2-BCQ. Body Checking Questionnaire; 3 - BSQ. Body Shape Questionnaire; 4 - EAT-26. Eating Attitudes Test - 26; $M=$ Média; $D P=$ Desvio-Padrão.

$* p<0,01$.

corresponde ao primeiro tercil da variação máxima do MBCQ (19 a 44 pontos), indicando baixa frequência de checagem corporal, uma vez que quanto maior a pontuação obtida maior é a frequência do comportamento.

\section{Análise Fatorial Exploratória (AFE)}

$\mathrm{O}$ teste de esfericidade de Bartlett foi significativo, $\chi^{2}(171)=3244,849, p<0,001$, e a medida KMO foi de 0,901 ; ambos indicaram que seria adequado prosseguir com a AFE do MBCQ. Pode-se notar que a solução fatorial encontrada explica $64,32 \%$ da variância total dos dados (Tabela 2). Estes foram nomeados de acordo com sua estrutura latente: Fator 1 (Checagem por comparação com outras pessoas [CCO]; eigenvalue $=8,37$ ), Fator 2 (Checagem muscular - uso do espelho [CME]; eigenvalue $=1,53$ ), Fator 3 (Checagem por avaliação externa [CAE]; eigenvalue $=1,29$ ), e Fator 4 (Checagem de gordura $[\mathrm{CG}]$; eigenvalue $=1,02)$. A consistência interna obtida de cada um dos quatro fatores foi satisfatória (Tabela 2).

Tabela 2

Cargas Fatoriais da AFE (com Rotação Varimax) e Porcentagem da Variância Explicada pelos Quatro Fatores do MBCQ

Itens
1. Checo a firmeza de meus braços para confirmar que não perdi nenhuma
massa muscular.

massa muscular.

2. Olho meus músculos abdominais - "tanquinho" - no espelho.

Fator $1 \quad$ Fator $2 \quad$ Fator $3 \quad$ Fator 4

3. Quando me olho no espelho, contraio os braços para confirmar a igualdade entre eles.

4. Comparo o tamanho de meus músculos com o de outras pessoas

0,238

(a)

0,359

0,687

0,123

0,171

0,359

$\mathbf{0 , 5 8 0}$

0,113

$-0,026$

0,357

0,722

0,162

0,049

0,689

0,300

0,178

0,153

5. Comparo minha "magreza" ou definição muscular com a de outras pessoas.

0,795

0,057

0,065

0,127

6. Comparo meus músculos com a dos atletas ou das celebridades.

0,718

0,336

0,197

$-0,082$

7. Comparo minha "magreza" ou definição muscular com a dos atletas ou das celebridades.

$\mathbf{0 , 7 5 9}$

$$
0,241
$$

0,187

$-0,081$

8. Peço a outras pessoas para tocarem em meus músculos para confirmar o tamanho e a firmeza deles.

0,167

$0,144 \quad \mathbf{0 , 8 6 3} \quad 0,008$

9. Peço a outras pessoas para comentarem sobre a definição ou o tamanho de meus músculos.

10. Belisco a gordura da minha barriga e costas (por exemplo, os pneuzinhos) para checar a minha "magreza".

\section{0}

11. Comparo minha "magreza" ou a definição de meus músculos peitorais a de outras pessoas.

12. Comparo o tamanho de meus músculos peitorais com o tamanho dos músculos de outras pessoas.

13. Comparo a largura de meus ombros com a largura dos ombros de outras pessoas.

0,

14. Contraio meus músculos peitorais diante do espelho para confirmar a igualdade entre eles.

$\begin{array}{llll}\mathbf{0 , 7 7 3} & 0,269 & 0,116 & 0,322 \\ \mathbf{0 , 7 6 0} & 0,333 & 0,129 & 0,291 \\ \mathbf{0 , 6 2 1} & 0,203 & 0,186 & 0,316 \\ 0,328 & \mathbf{0 , 7 0 7} & 0,125 & 0,155\end{array}$


Carvalho, P. H. B., Conti, M. A., Ribeiro, M. S., Amaral, A. C. S. \& Ferreira, M. E. C. (2014). Avaliação Psicométrica do Male Body Checking Questionnaire (MBCQ).

15. Contraio meus músculos diante do espelho a procura de linhas ou estriamentos neles.

\begin{tabular}{cccc}
0,206 & $\mathbf{0 , 5 5 2}$ & 0,109 & 0,440 \\
0,125 & $\mathbf{0 , 5 4 0}$ & 0,260 & 0,391 \\
0,210 & 0,193 & 0,109 & $\mathbf{0 , 7 7 2}$ \\
& & & \\
0,356 & $\mathbf{0 , 5 4 7}$ & 0,113 & 0,287 \\
& & & \\
0,253 & $\mathbf{0 , 7 0 7}$ & 0,079 & 0,287 \\
$\mathbf{4 4 , 0 6}$ & $\mathbf{8 , 0 8}$ & $\mathbf{6 , 7 9}$ & $\mathbf{5 , 3 9}$ \\
$\mathbf{0 , 9 0}$ & $\mathbf{0 , 8 8}$ & $\mathbf{0 , 7 6}$ & $\mathbf{0 , 7 1}$ \\
\hline
\end{tabular}

16. Meço meus músculos com uma fita métrica.

17. Aperto a gordura ou estico a pele do meu corpo para acentuar o músculo escondido pela gordura.

18. Checo o tamanho e a forma de meus músculos na maioria das superfícies espelhadas (por exemplo, nas janelas de carros, nas vitrines de lojas, nos espelhos, etc).

19. Belisco ou aperto meus músculos para confirmar o tamanho e a firmeza deles.

Porcentagem da variância explicada.

$\alpha$-Cronbach

0,90

Nota. Em destaque as cargas fatoriais indicando a qual fator pertence o item.

\section{Análise Fatorial Confirmatória (AFC)}

A AFC apresentou os seguintes valores de ajuste do modelo: $\chi^{2}$ significativo $\left[\chi^{2}(146)=562,14, p<0,01\right]$; CFI $(0,94)$; TLI $(0,94)$; IFI $(0,95)$; RMSEA igual a $0,099(90 \%$ $\mathrm{IC}=0,09-0,11 ; p<0,001)$, SRMR $(0,05)$ e AIC (Model $=650,14 \mathrm{e}$ Independence $=8696,86$ ).

$\mathrm{O}$ teste $\chi^{2}$ foi significativo, indicando diferença entre as covariâncias do modelo hipotético ideal e do modelo real. Os índices utilizados para ajuste do modelo real (CFI, TLI e IFI) são considerados adequados (Bentler \& Bonett, 1980), assim como o AIC, que obteve AIC Model menor do que o AIC Independence (Akaike, 1987). O valor de RMSEA foi marginal (Browne \& Cudeck, 1992), enquanto sua medida alternativa (SRMR) foi adequada (Kahn, 2006). Os dados, portanto, suportam a evidência de validade de construto do MBCQ.

\section{Validade Convergente}

O MBCQ associou-se moderada (Landis \& Kock, 1977) e significativamente $(p<0,01)$ com o BCQ e com o BSQ, $r=0,51$ e $r=0,35$, respectivamente (Tabela 1 ). Ou seja, quanto maior a frequência do comportamento de checagem corporal, maior é a insatisfação corporal do sujeito. Esta associação é esperada e deve ocorrer de acordo com Fairburn et al. (1999). O MBCQ também obteve associação com outra medida destinada a avaliação de comportamentos de checagem corporal. Essa associação já foi demonstrada por (Hildebrandt et al., 2010) no processo de validação inicial do instrumento.

Na Tabela 3, são apresentados os valores de correlação entre os escores dos fatores dos instrumentos MBCQ e BCQ. Observa-se que os instrumentos apresentam uma associação entre seus fatores, indicando que os mesmos apresentam similaridade no construto avaliado (validade convergente).

Tabela 3

Correlação entre os Fatores dos Instrumentos

\begin{tabular}{lcccccccc}
\hline \multicolumn{1}{c}{ Variáveis } & 1 & 2 & 3 & 4 & 5 & 6 & 7 & 8 \\
\hline 1. MBCQ (CCO) & - & & & & & & \\
2. MBCQ (CME) & $0,70^{*}$ & - & & & & & \\
3. MBCQ (CG) & $0,41^{*}$ & $0,39^{*}$ & - & & & & & \\
4. MBCQ (CAE) & $0,41^{*}$ & $0,51^{*}$ & $0,27^{*}$ & - & & & & \\
5. BCQ (OB) & $0,20^{*}$ & $0,25^{*}$ & 0,06 & $0,40^{*}$ & - & & \\
6. BCQ (ME) & $0,40^{*}$ & $0,49^{*}$ & 0,09 & $0,48^{*}$ & $0,40^{*}$ & - & \\
7. BCQ (CO) & $0,41^{*}$ & $0,35^{*}$ & 0,10 & $0,17 *$ & $0,25^{*}$ & $0,32^{*}$ & - & \\
8. MBCQ (IP) & $0,29 *$ & $0,28^{*}$ & 0,09 & $0,40^{*}$ & $0,58^{*}$ & $0,35^{*}$ & $0,38^{*}$ & - \\
\hline
\end{tabular}

Nota. MBCQ (CCO - checagem por comparação com outras pessoas; CME - checagem muscular - espelho; CG - checagem de gordura; $\mathrm{CAE}$ - checagem por avaliação externa); $\mathrm{BCQ}(\mathrm{OB}$ - checagem pela observação do corpo; $\mathrm{ME}$ - checagem por meio de medições de partes do corpo; $\mathrm{CO}$ - checagem por comparação entre o corpo do sujeito e os corpos dos outros; IP - busca por informações perceptivas).

$* p<0,01$. 


\section{Validade Discriminante}

A comparação entre o grupo com baixo e elevado escore para sintomas de TA (EAT-26) apresentou diferença estatisticamente significativa $(p=0,018)$ quanto ao MBCQ. Hildebrandt et al. (2010) relatam que a MBCQ é capaz de discriminar indivíduos com comportamentos de purgação e/ou de compulsão alimentar daqueles sem esses comportamentos. As evidências obtidas na presente pesquisa suportam a validade discriminante do MBCQ. Sujeitos com elevado escore de comportamentos de risco para os TA apresentaram maior frequência dos comportamentos de checagem corporal do que aqueles com baixo comportamento de risco.

\section{Reprodutibilidade}

Não houve diferença estatisticamente significativa $(p=0,26)$ na comparação entre os escores do MBCQ obtidos nos momentos 1 e 2, o que indica estabilidade do instrumento. O coeficiente de correlação intraclasse correspondeu a 0,87 e o $\alpha$-Cronbach obtido foi de 0,89 e 0,94 , nos momentos $1 \mathrm{e} 2$, respectivamente. Tais resultados sugerem que o MBCQ apresenta estabilidade e confiabilidade, quando aplicado em momentos diferentes (duas semanas de intervalo).

\section{Discussão}

Este estudo avaliou a estrutura fatorial, validade convergente e discriminante, e a reprodutibilidade do MBCQ. Foi testada, por meio da AFE, a estrutura do instrumento, que apresentou a existência de quatro fatores, com percentual de explicação da variância total adequado (superior a $60 \%$ ), similar ao obtido no processo de validade do instrumento original.

Os itens correspondentes a cada fator agruparam-se diferentemente do instrumento original, reunidos de acordo com a maneira como o comportamento de checagem é realizado: comparação com outras pessoas (por exemplo, "Comparo o tamanho de meus músculos com o de outras pessoas"); uso de superfície reflexiva (por exemplo, "Olho meus músculos abdominais - "tanquinho" - no espelho"); checagem por meio de "pinçamento" - gordura (por exemplo, "Aperto a gordura ou estico a pele do meu corpo para acentuar o músculo escondido pela gordura"); e pela avaliação do seu corpo por outras pessoas (por exemplo, "Peço a outras pessoas para comentar sobre a definição ou o tamanho de meus músculos").

No estudo de Hildebrandt et al. (2010), os itens são agrupados em seus fatores de forma que estes são representados não somente pela maneira como a checagem ocorre (por exemplo, comparação ou teste corporal), mas também se dividem pela área corporal em questão (por exemplo, geral - corpo como um todo; ou específica ombros e peitorais).

Segundo Beaton, Bombardier, Guillemin, e Ferraz (2000) e Sousa e Rojjanasrirat (2011), a adaptação transcultural de escalas requer uma adequação das mesmas a sua cultura local, e, dessa maneira, a forma de administração do instrumento, o número de itens e, ainda, os valores das propriedades psicométricas não precisam ser, necessariamente, idênticos. No entanto, os valores, tanto de validade quanto de reprodutibilidade, devem ser apresentados. $\mathrm{O}$ agrupamento de itens do MBCQ, obtidos nessa pesquisa, pode ser explicado pela forma como os indivíduos participantes dessa pesquisa compreenderam as questões propostas pelo instrumento.

$\mathrm{O}$ teste de esfericidade de Bartlett foi significativo e a medida KMO adequada, indicando na AFE, adequação dos dados para seguimento da análise fatorial. $\mathrm{O}$ teste Qui-quadrado, realizado na AFC, foi significativo, demonstrando diferença entre as covariâncias do modelo hipotético ideal e do modelo real, ou seja, pobre ajuste. No entanto, segundo Jackson, Gillaspy e Purc-Stephenson (2009) e Kahn (2006), o Qui-quadrado apresenta grande sensibilidade ao tamanho amostral, o que, de modo recorrente, resulta em significância estatística, mas, no entanto, não indica, necessariamente, pobre ajuste do modelo. Para os autores, bons índices de ajuste atestam para uma adequação do modelo proposto e indicam uma parcimônia do mesmo. Desta maneira, recorreu-se a análise de alguns índices de ajuste do modelo proposto (real), como medida alternativa de teste do modelo e verificação da adequação do mesmo.

Os índices obtidos na AFC relativos ao modelo de ajuste (CFI, TLI e IFI) são considerados adequados (Bentler $\&$ Bonett, 1980). O AIC Model foi menor do que o AIC Independence, indicando bom ajuste do modelo (Akaike, 1987). Baseado nesses resultados é possível confirmar que o modelo de solução fatorial encontrado (modelo real) é adequado em relação ao modelo hipotético ideal, ainda que o teste Qui-quadrado tenha apresentado significância estatística.

A SRMR foi adequada (bom ajuste). No entanto, a RMSEA apresentou valor marginal. Browne e Cudeck (1992) sugerem que valores abaixo de 0,05 são excelentes, entre 0,05 e 0,08 adequados, entre 0,08 e 1 (um) marginais, e, acima de 1 (um) - pobre ajuste. Desta maneira, o modelo de solução fatorial encontrado (modelo real) foi considerado adequado em virtude da utilização da SRMR como medida alternativa à RMSEA (Kahn, 2006).

A partir das análises fatoriais aqui identificadas, o MBCQ apresentou dois fatores (Fatores 3 e 4 - ver Tabela 2) formados, cada um, por apenas dois itens. Fabrigar, Wegener, MacCallum e Strahan (1999) recomendam um mínimo de três itens por fator para a análise das propriedades psicométricas. No entanto, o percentual de explicação da variância do instrumento e os bons índices de ajuste obtidos pelo modelo proposto suportam a adequada estrutura fatorial obtida. Sendo assim, optou-se pela manutenção dos Fatores 3 e 4, pois estes contribuem para a análise do construto (checagem corporal).

Hildebrandt et al. (2010) sugeriram que o fato de homens e mulheres apresentarem preocupações diferentes quanto ao corpo (por exemplo, muscularidade versus 
Carvalho, P. H. B., Conti, M. A., Ribeiro, M. S., Amaral, A. C. S. \& Ferreira, M. E. C. (2014). Avaliação Psicométrica do Male Body Checking Questionnaire (MBCQ).

magreza), bem como distintos comportamentos para controle do peso corporal (por exemplo, uso de esteroides anabolizantes e prática excessiva de exercício físico versus uso de laxantes e restrição alimentar), seria refletido em uma associação inversamente proporcional entre o MBCQ e BCQ. Tal hipótese não foi confirmada no estudo original de validação do MBCQ, bem como no presente estudo. Hildebrandt et al. (2010) asseveram que, apesar das diferenças entre os instrumentos, esses apresentam uma unidade: a avaliação do comportamento de checagem corporal.

Em estudo sobre o impacto do sexo do respondente sobre os escores obtidos no MBCQ e BCQ, Alfano, Hildebrandt, Bannon, Walker, e Walton (2011) encontraram evidência de que 10 itens apresentam correspondência entre os instrumentos. Segundo os autores, ao menos esses itens poderiam ser utilizados em amostras tanto de homens como de mulheres, sem prejuízo à validade das informações obtidas. No entanto, é possível que esses, quando utilizados em separado, não reflitam da mesma maneira o construto checagem corporal. Para tanto é necessária uma análise mais aprofundada acerca da validade desses itens.

Os dados de nossa pesquisa reforçam os achados de Alfano et al. (2011) e Hildebrandt et al. (2010). Observou-se correlação entre os fatores dos instrumentos MBCQ e BCQ, assim como entre os fatores de cada instrumento entre si (Tabela 3). Esses achados reforçam também o fato de os instrumentos avaliarem formas específicas de comportamentos de checagem que, em conjunto, conferem uma unidade de avaliação: checagem corporal.

O MBCQ obteve correlação significativa com outra medida, o BSQ, indicando que quanto maior a insatisfação corporal, maiores são os comportamentos de checagem corporal (evidência de validade convergente). Esta associação é destacada por Fairburn et al. (1999), que sugerem uma correlação entre os comportamentos de checagem corporal e os aspectos psicopatológicos dos TA, como a insatisfação com o corpo.

$\mathrm{O}$ instrumento em análise comprovou sua capacidade em diferenciar os indivíduos com baixo e alto comportamento alimentar inadequado quanto aos comportamentos de checagem corporal (evidência de validade discriminante). Hildebrandt et al. (2010) atestam que o MBCQ é um instrumento adequado para o rastreamento de indivíduos com sintomas de TA, o que pode ser confirmado pelos dados dessa pesquisa.

Este estudo apresenta algumas limitações. Primeiro, foi utilizada uma amostra por conveniência, fato que compromete a generalização dos dados de validade e reprodutibilidade para outras amostras. No entanto, esta forma de seleção da amostra é usual em estudos de avaliação psicométrica. Sugere-se que a consistência interna do instrumento seja avaliada em outros estudos envolvendo o MBCQ. Segundo, não houve controle, além da não inclusão de graduandos dos cursos de Educação Física e Nutrição, relativos à saúde dos participantes ou informações relativas à sua saúde geral. A presença de alguns transtornos mentais, como por exemplo, o transtorno dismórfico corporal, pode interferir no construto em avaliação. Terceiro, a média de idade dos participantes correspondeu à faixa etária de adultos jovens (18 aos 30 anos), não sendo possível atestar sua validade para amostras de indivíduos de meia-idade e idosos, bem como entre adolescentes. Cumpre ressaltar que, na população masculina, os TA se desenvolvem mais tardiamente do que nas mulheres, sendo mais evidente em indivíduos entre os 18 e 26 anos (Braun, Sunday, Huang, \& Halmi, 1999), faixa etária média compreendida neste estudo. Por fim, para teste de evidência de validade discriminante foi utilizada a análise dimensional do EAT-26, sugerida por Orbitello et al. (2006) para grupos não clínicos, em detrimento à comparação destes indivíduos com aqueles corretamente diagnosticados de TA. Embora em crescimento, a prevalência de TA na população masculina ainda é baixa, o que dificulta a coleta de um número representativo de indivíduos. Para obter tal representatividade amostral seria necessário um estudo multicêntrico, o que aumentaria o tempo e custo da pesquisa.

A despeito destas limitações, este estudo apresenta resultados empíricos de bons indicadores de validade e de reprodutibilidade da versão brasileira do MBCQ (Carvalho et al., 2012) para avaliação da checagem corporal em jovens do sexo masculino no Brasil, acrescentando uma importante escala para a investigação clínica e epidemiológica. Ressalta-se, no entanto, que a versão brasileira apresenta solução fatorial diferente da versão original do MBCQ. Estudos transculturais envolvendo comparação entre populações, além daqueles destinados à adaptação de instrumentos e verificação de suas propriedades psicométricas, devem ser realizados, no intuito de ampliar o conhecimento sobre comportamentos de checagem corporal, sua modulação e influência sobre o possível tratamento de indivíduos.

\section{Referências}

Akaike, H. (1987). Factor analysis and AIC. Psychometrika, 52, 317-332. doi:10.1007/BF02294359

Alfano, L., Hildebrandt, T., Bannon, K., Walker, C., \& Walton, K. E. (2011). The impact of gender on the assessment of body checking behavior. Body Image, 8, 20-25. doi:10.1016/j. bodyim.2010.09.005

American Psychiatric Association. (1994). DSM-IV: Diagnostic and statistical manual of mental disorders $\left(4^{\text {th }}\right.$ ed. $)$. Washington, DC: Author.

Beaton, D. E., Bombardier, C., Guillemin, F., \& Ferraz, M. B. (2000). Guidelines for the process of cross-cultural adaptation of self-report measures. Spine, 25, 3186-3191. doi:10.1097/00007632-200012150-00014

Bentler, P. M., \& Bonett, D. G. (1980). Significance tests and goodness of fit in the analysis of covariance structures. Psychological Bulletin, 88, 588-606. doi:10.1037/00332909.88.3.588

Bosi, M. L. M., Luiz, R. R., Uchimura, K. Y., \& Oliveira, F. P. (2008). Comportamento alimentar e imagem corporal entre estudantes de educação física. Jornal Brasileiro de Psiquiatria, 57, 28-33. doi:10.1590/S0047-20852008000100006 
Braun, D. L., Sunday, S. R., Huang, A., \& Halmi, K. (1999). More male seek treatment for eating disorders. International Journal of Eating Disorders, 25, 415-424. doi:10.1002/(SICI)1098108X(199905)25:4<415::AID-EAT6>3.0.CO;2-B

Browne, M. W., \& Cudeck, R. (1992). Alternative ways of assessing model fit. Sociological Methods \& Research, 21(2), 230-258.

Campana, A. N. N. B. (2007). Tradução, adaptação transcultural e validação do "Body Image Avoidance Questionnaire (BIAQ)" e do "Body Checking Questionnaire (BCQ)" para a língua portuguesa no Brasil (Dissertação de mestrado em Educação Física, Faculdade de Educação Física, Universidade Estadual de Campinas, SP, Brasil).

Carvalho, P. H. B., Conti, M. A., Cordás, T. A., \& Ferreira, M. E. C. (2012). Portuguese (Brazil) translation, semantic equivalence and internal consistency of the Male Body Checking Questionnaire (MBCQ). Revista de Psiquiatria Clínica, 39, 74-75. doi:10.1590/S0101-60832012000200006

Cash, T. F., \& Smolak, L. (Eds.). (2011). Body image: A handbook of science, practice, and prevention. New York: Guilford Press.

Cooper, P., Taylor, M. J., Cooper, Z., \& Fairburn, C. G. (1987). The development and validation of the Body Shape Questionnaire. International Journal of Eating Disorders, 6, 485-494. doi:10.1002/1098-108X(198707)6:4<485::AIDEAT2260060405>3.0.CO;2-O

Di Pietro, M., \& Silveira, D. X. (2009). Internal validity, dimensionality and performance of the Body Shape Questionnaire in a group of Brazilian college students. Revista Brasileira de Psiquiatria, 31, 21-24. doi:10.1590/S151644462008005000017

Exterkate, C. C., Vriesendorp, P. F., \& De-Jong, C. A. J. (2009). Body attitudes in patients with eating disorders at presentation and completion of intensive outpatient day treatment. Eating Behaviors, 10, 16-21. doi:10.1016/j.eatbeh.2008.10.002

Fabrigar, L. R., Wegener, D. T., MacCallum, R. C., \& Strahan, E. J. (1999). Evaluating the use of exploratory factor analysis in psychological research. Psychological Methods, 4, 272-299. doi:10.1037/1082-989X.4.3.272

Fairburn, C. G. (2008). Cognitive behavior therapy and eating disorders. New York: Guilford Press.

Fairburn, C. G., Shafran, R., \& Cooper, Z. (1999). A cognitive behavioural theory of anorexia nervosa. Behaviour Research and Therapy, 37, 1-13. doi:10.1016/S0005-7967(98)00102-8

Fiates, G. M. R., \& Salles, R. K. (2001). Fatores de risco para o desenvolvimento de distúrbios alimentares: Um estudo em universitárias. Revista de Nutrição, 14, 3-6. doi:10.1590/ S1415-52732001000400001

Garner, D. M., Olmsted, M. P., Bohr, Y., \& Garfinkel, P. E. (1982). The Eating Attitudes Test: Psychometric features and clinical correlates. Psychological Medicine, 12, 871-878. doi:10.1017/S0033291700049163

Hair, J. F., Tatham, R. L., Anderson, R. E., \& Black, W. (2005). Análise multivariada de dados. Porto Alegre, RS: Bookman.

Hildebrandt, T., Walker, D. C., Alfano, L., Delinsky, S., \& Bannon, K. (2010). Development and validation of a Male Specific Body Checking Questionnaire. International Journal of Eating Disorders, 43, 77-87. doi:10.1002/eat.20669

Jackson, D. L., Gillaspy, J. A., \& Purc-Stephenson, R. (2009). Reporting practices in confirmatory factor analysis: An overview and some recommendations. Psychological Methods, 14, 6-23. doi:10.1037/a0014694
Kachani, A. T., Barbosa, A. L. R., Brasiliano, S., Cordás, T. A., Hochgraf, P. B., \& Conti, M. A. (2011). Portuguese (Brazil) translation, cross-cultural adaptation and content validity of Body Checking Cognitions Scale - BCCS. Revista de Psiquiatria Clínica, 38, 13-18. doi:10.1590/S010160832011000100004

Kachani, A. T., Hochgraf, P. B., Brasiliano, S., Barbosa, A. L. R., Cordás, T. A., \& Conti, M. A. (2011). Psychometric evaluation of the Body Checking and Avoidance Questionnaire - BCAQ - adapted to Brazilian Portuguese. Eating and Weight Disorders, 16, 293-299. doi:10.1007/BF03327477

Kahn, J. H. (2006). Factor analysis in counseling psychology research, training, and practice: Principles, advances, and applications. The Counseling Psychologist, 34, 684-718. doi:10.1177/0011000006286347

Kline, R. B. (1998). Principles and practice of structural equation modeling. New York: The Guilford Press.

Landis, J. R., \& Kock, G. G. (1977). The measurement of observer agreement for categorical data. Biometrics, 33, 159-174. doi: $10.2307 / 2529310$

Mountford, V., Haase, A., \& Waller, G. (2006). Body checking in the eating disorders: Associations between cognitions and behaviors. International Journal of Eating Disorders, 39, 708-715. doi:10.1002/eat.20279

Murray, S. B., Rieger, E., Hildebrandt, T., Karlov, L., Russell, J., Boon, E., ...Touyz, S. W. (2012). A comparison of eating, exercise, shape, and weight related symptomatology in males with muscle dysmorphia and anorexia nervosa. Body Image, 9, 193-200. doi:10.1016/j.bodyim.2012.01.008

Nunes, M. A., Camey, S., Olinto, M. T. A., \& Mari, J. J. (2005). The validity and 4-year test-retest reliability of the Brazilian version of the Eating Attitudes Test-26. Brazilian Journal of Medical and Biological Research, 38, 1655-1662. doi:10.1590/S0100-879X2005001100013

Orbitello, B., Ciano, R., Corsaro, M., Rocco, P. L., Taboga, C., Tonutti, L., ...Balestrieri, M. (2006). The EAT-26 as screening instrument for clinical nutrition unit attenders. International Journal of Obesity, 30, 977-981. doi:10.1038/ sj.ijo.0803238

Pope, H. G., Phillips, K. A., \& Olivardia, R. (2000). The Adonis complex: The secret crisis of male body image obsession. New York: Free Press.

Reas, D. L., Grilo, C. M., Masheb, R. M., \& Wilson, G. T. (2005). Body checking and avoidance in overweight patients with binge eating disorder. International Journal of Eating Disorders, 37, 342-346. doi:10.1002/eat.20092

Reas, D. L., Whisenhunt, B. L., Netemeyer, R., \& Williamson, D. A. (2002). Development of the Body Checking Questionnaire: A self-report measure of body checking behaviors. International Journal of Eating Disorders, 31, 324-333. doi:10.1002/eat.10012

Rosen, J. C., Reiter, J., \& Orosan, P. (1995). Cognitivebehavioral body image therapy for body dysmorphic disorder. Journal of Consulting and Clinical Psychology, 3, 263-269. doi:10.1037/0022-006X.63.2.263

Shafran, R., Fairburn, C. G., Robinson, P., \& Lask, B. (2004). Body checking and its avoidance in eating disorders. International Journal of Eating Disorders, 35, 93-101. doi:10.1002/ eat. 10228

Shafran, R., Lee, M., Payne, E., \& Fairburn, C. G. (2007). An experimental analysis of body checking. Behaviour Research and Therapy, 45, 113-121. doi:10.1016/j.brat.2006.01.015 
Carvalho, P. H. B., Conti, M. A., Ribeiro, M. S., Amaral, A. C. S. \& Ferreira, M. E. C. (2014). Avaliação Psicométrica do Male Body Checking Questionnaire (MBCQ).

Sousa, V. D., \& Rojjanasrirat, W. (2011). Translation, adaptation and validation of instruments or scales for use in cross-cultural health care research: A clear and user-friendly guideline. Journal of Evaluation in Clinical Practice, 17, 268-274. doi:10.1111/j.1365-2753.2010.01434.x

Stice, E., \& Shaw, H. E. (2002). Role of body dissatisfaction in the onset and maintenance of eating pathology: A synthesis of research findings. Journal of Psychosomatic Research, 53, 985-993. doi:10.1016/S0022-3999(02)00488-9

Streiner, D. L. (2003). Starting at the beginning: An introduction to coefficient alpha and internal consistency. Journal of Personality Assessment, 80, 99-103. doi:10.1207/ S15327752JPA8001 18

Thompson, J. K. (2004). The (mis) measurement of body image: Ten strategies to improve assessment for applied and research purposes. Body Image, 1, 7-14. doi:10.1016/S17401445(03)00004-4

Thompson, J. K., \& Cafri, G. (2007). The muscular ideal: Psychological, social, and medical perspectives. Washington, DC: American Psychological Association.

Williamson, D. A., Muller, S. L., Reas, D. L., \& Thaw, J. M. (1999). Cognitive bias in eating disorders: Implications for theory and treatment. Behavior Modification, 23, 556-577. doi:10.1177/0145445599234003 